\title{
Fitting DVCS at NLO and beyond
}

\author{
K. Kumerički ${ }^{1}$, D. Müller ${ }^{2}$, K. Passek-Kumerički ${ }^{3 \dagger}$ \\ ${ }^{1}$ Department of Physics, Faculty of Science, University of Zagreb, Croatia \\ ${ }^{2}$ Institut für Theoretische Physik II, Ruhr-Universität Bochum, Germany \\ ${ }^{3}$ Theoretical Physics Division, Rudjer Bošković Institute, Croatia
}

\begin{abstract}
We outline the twist-two analysis of deeply virtual Compton scattering within the $S O(2,1)$ partial wave expansion of the amplitude, represented as a Mellin-Barnes integral. The complete next-to-leading order results, including evolution, are obtained in the $\overline{\mathrm{MS}}$ and a conformal factorization scheme. Within the latter, exploiting conformal symmetry, the radiative corrections are evaluated up to next-to-nextto-leading order. Using a new proposed parameterization for GPDs, we study the convergence of perturbation theory and demonstrate that our formalism is suitable for a fitting procedure of DVCS observables.
\end{abstract}

\section{Introduction}

The proton structure has been widely explored in inclusive measurements, mainly, in deeply inelastic lepton-proton scattering (DIS). Here the scattering essentially occurs due to the exchange of a virtual boson (photon) on a single parton, and so one can access parton distribution functions (PDFs). This universal, however, convention-dependent functions $q_{a}(x)$ are interpreted as probabilities that partons of certain flavour $a$ will be found with given longitudinal momentum fraction $x$. Since the PDFs are naturally defined in a translation invariant manner, they do not carry information about the transversal distribution of partons. Some information about transversal degrees of freedom can be obtained from elastic lepton-proton scattering. The electromagnetic form factors $F_{1,2}(t)$ are Fourier transforms of the electric and magnetic charge distribution in nucleon, and can be, e.g., in the infinite momentum frame, interpreted as probability that partons are found at some transversal distance $\mathbf{b}$ from the center-of-mass. However, one can not assume that a realistic probability distribution of partons, given by a two-variable function $q(x, \mathbf{b})$ is simply a direct product, i.e., $q(x) \otimes q(\mathbf{b})$, of two probability functions. Rather it is anticipated that longitudinal and transversal degrees of freedom have a cross talk, e.g., as $x$ gets bigger partons carry more of the nucleon longitudinal momentum and are expected to be closer to the proton center, and thus the $\mathbf{b}$ dependence in $q(x, \mathbf{b})$ should become narrower with increasing $x$.

The three dimensional distribution of partons in the nucleon can be addressed within more general objects, called generalized parton distributions (GPDs) [1,2]. Such distributions can be revealed by analyzing hard exclusive leptoproduction of mesons or photon. The former processes are perhaps theoretically more problematic to describe but offer a direct view into individual flavour GPDs. To the latter one the deeply virtual Compton scattering (DVCS) process

\footnotetext{
${ }^{\dagger}$ speaker
} 


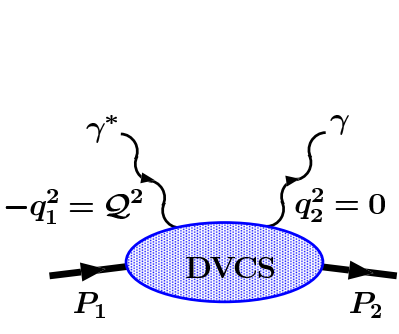

a)

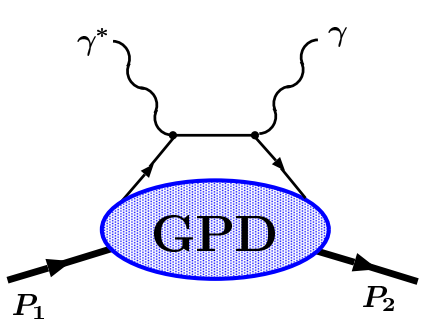

b)

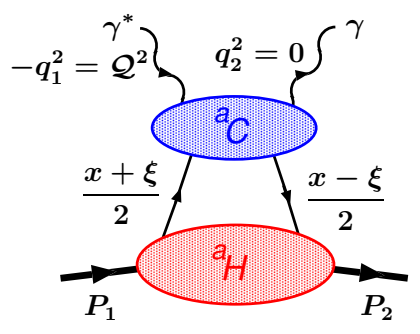

c)

Fig. 1: a) DVCS. b) Leading-order perturbative contribution to DVCS. c) Factorization (to all orders in $\alpha_{s}$ ) on an example of special parity-even, helicity conserving contribution ${ }^{a} \mathcal{H}(5)$.

contributes, where one photon has a large virtuality. DVCS is theoretically considered as the cleanest probe of GPDs, however, here only certain flavour combinations of GPDs appear.

The non-forward Compton scattering process is described by the Compton tensor

$$
T_{\mu \nu}(q, P, \Delta)=\frac{i}{e^{2}} \int d^{4} x e^{i x \cdot q}\left\langle P_{2}, S_{2}\left|T j_{\mu}(x / 2) j_{\nu}(-x / 2)\right| P_{1}, S_{1}\right\rangle,
$$

where $q=\left(q_{1}+q_{2}\right) / 2, P=P_{1}+P_{2}$ and $\Delta=P_{2}-P_{1}$. The generalized Bjorken limit corresponds to $Q^{2}=-q^{2} \rightarrow \infty$ with the scaling variables

$$
\xi=\frac{Q^{2}}{P \cdot q}, \quad \eta=-\frac{\Delta \cdot q}{P \cdot q},
$$

and the momentum transfer squared $\Delta^{2}$ being fixed. Note that in the forward case, i.e., $\Delta \rightarrow 0$, the hadronic DIS tensor $W_{\mu \nu}$ is related to the forward Compton scattering tensor by the optical theorem $W_{\mu \nu}=\Im \mathrm{m} T_{\mu \nu}(q, P=2 p, \Delta=0) /(2 \pi)$, where $p=P_{1}=P_{2}$ and $\xi \rightarrow x_{B j}$.

In DVCS - Fig.1a - the virtuality of the incoming photon $\mathcal{Q}^{2}=-q_{1}^{2}$ is large while the final photon is on-shell. The skewness parameter $\eta$ and the Bjorken-like scaling parameter $\xi$ are then equal to twist-two accuracy, i.e., $\eta=\xi+\mathcal{O}\left(1 / \mathcal{Q}^{2}\right)$. In the generalized Bjorken limit, similarly as for DIS structure functions, the amplitude factorizes into long- and short-distance contributions (Fig.1b and c): short-distance physics is perturbatively calculable Compton scattering on a parton, while long-distance physics is encoded in an non-perturbative amplitude for a parton being emitted and later reabsorbed by the nucleon. The latter amplitude is called GPD.

The GPDs with even parity, we are considering here, are defined as

$$
\begin{aligned}
{ }^{q} F\left(x, \eta, \Delta^{2}\right) & =\left.\int \frac{d z^{-}}{2 \pi} e^{i x P^{+} z^{-}}\left\langle P_{2}\left|\bar{q}(-z) \gamma^{+} q(z)\right| P_{1}\right\rangle\right|_{z^{+}=0, \mathbf{z}_{\perp}=\mathbf{0}} \\
{ }^{G} F\left(x, \eta, \Delta^{2}\right) & =\left.\frac{4}{P^{+}} \int \frac{d z^{-}}{2 \pi} e^{i x P^{+} z^{-}}\left\langle P_{2}\left|G_{a}^{+\mu}(-z) G_{a \mu}{ }^{+}(z)\right| P_{1}\right\rangle\right|_{z^{+}=0, \mathbf{z}_{\perp}=\mathbf{0}},
\end{aligned}
$$

and similar for odd parity. Furthermore, it is convenient to decompose GPDs,

$$
{ }^{a} F=\frac{\bar{u}\left(P_{2}\right) \gamma^{+} u\left(P_{1}\right)}{P^{+}}{ }^{a} H+\frac{\bar{u}\left(P_{2}\right) i \sigma^{+\nu} u\left(P_{1}\right) \Delta_{\nu}}{2 M P^{+}}{ }^{a} E, \quad a=q, G .
$$


into helicity conserving and non-conserving generalized form factors. The Compton tensor (1) is analogously decomposed into Compton form factors with corresponding parity and helicity properties. In the forward limit $(\Delta \rightarrow 0)$ GPDs reduce to PDFs. Together with sum rules, e.g., relating GPDs to electromagnetic form factors, this provides constraints that are important for GPD modelling. But as they do not constrain much the skewness $(\eta)$ dependence, modelling is still a difficult problem. One guiding feature is a polynomiality property of GPDs: $n$-th (Mellin) moment of GPD is even polynomial in $\eta$ of order $n$ or $n \pm 1$. Similar polynomiality will be obeyed also for conformal moments which are just linear combination of Mellin moments. The usefulness of the GPDs has also been widely realized in connection with the spin problem, since they encode the angular momentum carried by the individual parton species, as explicated by the Ji's sum rule [2]. For recent detailed account of GPDs and their properties, we refer to [3].

In the following we outline the perturbative QCD approach to DVCS that is based on the conformal partial wave expansion, represented as Mellin-Barnes integral, as described in Ref. [4,5]. Here, as in Refs. [5-7], we concentrate on the dominant Compton form factor $\mathcal{H}$ corresponding to parity-even helicity-conserving GPD $H$. For simplicity, we present only the results for the singlet part which is dominant for the kinematics of collider experiments.

\section{Deeply virtual Compton scattering}

Besides DVCS the Bethe-Heitler brehmstrahlung process contributes to the measured hard photon leptoproduction off a proton. The Bethe-Heitler amplitude, known in leading order of the QED fine structure constant, is expressed in terms of the known electromagnetic form-factors. Generally, there are two types of DVCS experiments: collider experiments and fixed target experiments. The former usually provide information in the phase space $10^{-4} \lesssim \xi \lesssim 10^{-1}$ and $1 \mathrm{GeV}^{2} \lesssim \mathcal{Q}^{2} \lesssim 100 \mathrm{GeV}^{2}$, and such are $\mathrm{H} 1$ and ZEUS experiments at HERA. The main observables here are total and differential DVCS cross sections, however, also the beam charge asymmetry is feasible. For the fixed target experiments, such as Hall A, Hall B (CLAS) at JLAB, and HERMES at DESY, the interference term can be more easily accessed via single beam, target spin and beam charge (HERMES) asymmetries, while the investigated phase space covers the so-called valence quark region, i.e., $0.05 \lesssim \xi \lesssim 0.3$ within $1 \mathrm{GeV}^{2} \lesssim \mathcal{Q}^{2} \lesssim 10 \mathrm{GeV}^{2}$.

One can express $\mathcal{H}$ as a convolution (Fig.1c) over the longitudinal momentum fraction $x$

$$
{ }^{a} \mathcal{H}\left(\xi, \Delta^{2}, \mathcal{Q}^{2}\right)=\int \mathrm{d} x{ }^{a} C\left(x, \xi, \mathcal{Q}^{2} / \mu^{2}\right){ }^{a} H\left(x, \eta=\xi, \Delta^{2}, \mu^{2}\right),
$$

where $\mu^{2}$ is a factorization scale that separates short- and long-distance dynamics often taken $\mu^{2}=\mathcal{Q}^{2}$. Here the index $a \in\{\mathrm{NS}, \mathrm{S}(\Sigma, G)\}$ denotes either non-singlet or singlet parts, where to latter both quarks $(\Sigma)$ and gluons $(G)$ contribute.

The coefficient function $C^{a}$ is a perturbative quantity which describes $q \gamma^{*} \rightarrow q \gamma$ and $g \gamma^{*} \rightarrow g \gamma$ subprocesses. The well known leading-order (LO) contribution to $C^{a}$ is actually a pure QED process (Fig. 1b). The next-to-leading order (NLO) contribution - the first order in $\alpha_{s}$ - has been calculated by various groups [8]. Obviously, to stabilize the perturbation series and investigate its convergence one needs the second order in $\alpha_{s}$, i.e., next-to-next-to-leading order (NNLO) contributions. The importance of NNLO in singlet case is amplified by the fact that at LO photons scatter only off charged partons, whereas gluons start contributing at NLO. 
The GPDs are intrinsically non-perturbative quantities whose form at some initial scale has to be deduced by some non-perturbative methods (lattice calculation, fit to data etc.). The evolution to the factorization scale of interest is governed by perturbation theory, cf. [5, 7] for LO examples. The anomalous dimensions of non-diagonal operators were calculated up to NLO [9]. Still, NLO evolution is numerically not easy to implement and has been investigated beyond NLO only recently, using the below explained procedure [5].

Instead of using the convolution form (5) one can equivalently use the sum over the conformal moments, and the singlet contribution takes then the form

$$
\mathrm{S}_{\mathcal{H}}\left(\xi, \Delta^{2}, \mathcal{Q}^{2}\right)=2 \sum_{j=0}^{\infty} \xi^{-j-1} \boldsymbol{C}_{j}\left(\mathcal{Q}^{2} / \mu^{2}, \alpha_{s}(\mu)\right) \boldsymbol{H}_{j}\left(\eta=\xi, \Delta^{2}, \mu^{2}\right),
$$

where $\boldsymbol{C}_{j}=\left({ }^{\Sigma} C_{j},{ }^{G} C_{j}\right)$ and $\boldsymbol{H}_{j}=\left({ }^{{ }} H_{j},{ }^{G} H_{j}\right)$ are conformal moments. They are analogous to common Mellin moments used in DIS but the integral kernel $x^{j}$ is replaced by Gegenbauer polynomials $C_{j}^{3 / 2}(x)$ and $C_{j}^{5 / 2}(x)$, which are solutions of LO evolutional equations for quarks and gluons, respectively. Unfortunately, the series (6) only converges in the unphysical region. Hence, it is necessary to resum this series, e.g., by means of the Mellin-Barnes integral [5]

$$
\mathrm{S}_{\mathcal{H}}\left(\xi, \Delta^{2}, \mathcal{Q}^{2}\right)=\frac{1}{2 i} \int_{c-i \infty}^{c+i \infty} d j \xi^{-j-1}\left[i+\tan \left(\frac{\pi j}{2}\right)\right] \boldsymbol{C}_{j}\left(\mathcal{Q}^{2} / \mu^{2}, \alpha_{s}(\mu)\right) \boldsymbol{H}_{j}\left(\xi, \Delta^{2}, \mu^{2}\right) .
$$

The advantages of using conformal moments, i.e., Mellin-Barnes representation, are manifold. It allows for an efficient and stable numerical treatment, it enables a simple inclusion of evolution, and it opens a new door for interesting modelling of GPDs. Finally, by making use of conformal operator product expansion (OPE) and known NNLO DIS results, it enables the assessment of NNLO contributions.

\section{Conformal approach to DVCS beyond NLO}

Neither Wilson coefficients nor anomalous dimensions are calculated (only so-called quark bubble insertions were partly evaluated) in non-forward kinematics at NNLO. To access the NNLO of non-forward Compton scattering, we use the conformal approach, making it possible to calculate relevant objects using only diagonal results of forward Compton scattering, i.e., DIS.

DVCS belongs to a class of two-photon processes (DIS, DVCS, two-photon production of hadronic states $\ldots$ ) calculable by means of the OPE, $T_{\mu \nu}(q, P, \Delta) \rightarrow C_{j} O_{j}$, for which the use of generalized Bjorken kinematics and conformal symmetry enables a unified description. While massless QCD is conformally invariant at tree level, this invariance is broken at the loop level since renormalization introduces a mass scale, leading to the running of the coupling constant $(\beta \neq 0)$. Assuming the existence of a non-trivial fixed point $\alpha_{s}^{*}$, i.e., $\beta\left(\alpha_{s}^{*}\right)=0$, the conformal OPE (COPE) prediction for Wilson coefficients in general kinematics reads [10]

$$
C_{j}\left(\alpha_{s}^{*}\right)=c_{j}\left(\alpha_{s}^{*}\right)_{2} F_{1}\left(\begin{array}{c}
\left(2+2 j+\gamma_{j}\left(\alpha_{s}^{*}\right)\right) / 4,\left(4+2 j+\gamma_{j}\left(\alpha_{s}^{*}\right)\right) / 4 \\
\left(5+2 j+\gamma_{j}\left(\alpha_{s}^{*}\right)\right) / 2
\end{array} \mid \frac{\eta^{2}}{\xi^{2}}\right)\left(\frac{\mu^{2}}{Q^{2}}\right)^{\gamma_{j}\left(\alpha_{s}^{*}\right) / 2} .
$$



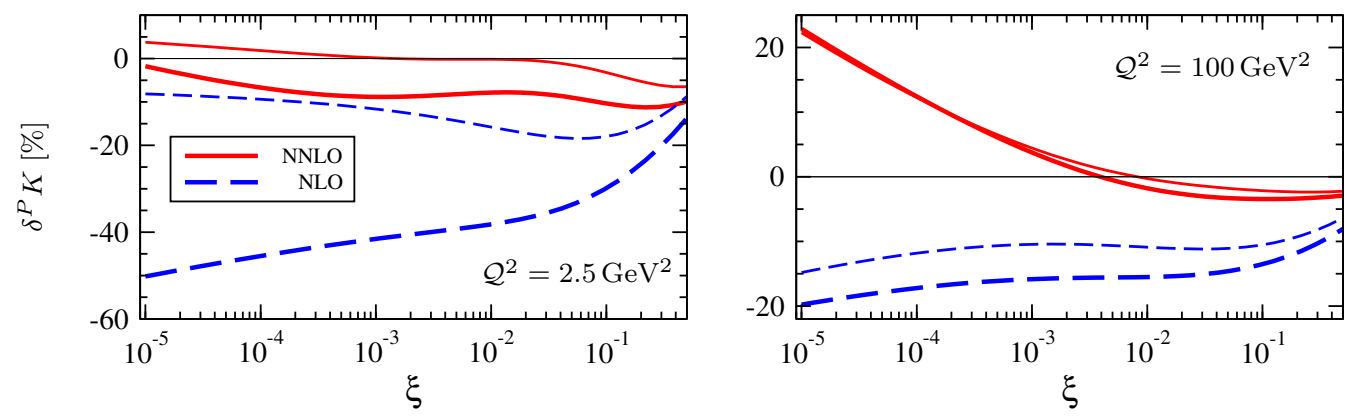

Fig. 2: Relative NLO and NNLO corrections (12) in the $\overline{\mathrm{CS}}$ scheme $\left(\Delta^{2}=0.25 \mathrm{GeV}^{2}\right.$, input scale $\left.\mathcal{Q}_{0}^{2}=2.5 \mathrm{GeV}^{2}\right)$. Thick [thin] lines - "hard" ["soft"] gluon: $N_{G}=0.4, \alpha_{G}(0)=\alpha_{\Sigma}(0)+0.05\left[N_{G}=0.3, \alpha_{G}(0)=\alpha_{\Sigma}(0)-0.02\right]$.

For $\eta=0$ equation (8) reduces to the DIS Wilson coefficients $C_{j} \rightarrow c_{j}$ and thus fixes the normalization $c_{j}$. The choice $\eta=\xi$ corresponds to DVCS in the conformal limit. The anomalous dimensions governing the evolution are diagonal and the same as in DIS.

For a general factorization scheme, e.g., the $\overline{\mathrm{MS}}$ scheme, the conformal symmetry breaking occurs also due to the renormalization of the composite operators and causes the appearance of non-diagonal anomalous dimensions $\gamma_{j k}=\delta_{j k} \gamma_{j}+\gamma_{j k}^{\mathrm{ND}}$. This induces a mixing of of both operators, i.e., GPDs, and Wilson coefficients under evolution. In the kinematical forward limit $(\eta=0)$ the diagonal evolution equations are again obtained, i.e., the DIS case corresponds to the COPE result. For DVCS, e.g., evaluated in the $\overline{\mathrm{MS}}$ scheme, there appear also conformal symmetry breaking terms which are not proportional to $\beta$, i.e., the non-diagonal terms survive.

The non-diagonal terms of anomalous dimensions encountered in $\overline{\mathrm{MS}}$ scheme can be removed by a finite renormalization [10], i.e, by a specific choice of the factorization scheme $C^{\overline{\mathrm{MS}}} O^{\overline{\mathrm{MS}}}=C^{\overline{\mathrm{MS}}} B B^{-1} O^{\overline{\mathrm{MS}}}=C^{\overline{\mathrm{CS}}} O^{\overline{\mathrm{CS}}}$. In this new scheme, called conformal subtraction $(\overline{\mathrm{CS}})$ scheme, all non-diagonal terms are "pushed" to the $\beta$ proportional part. $\gamma_{j k}^{\overline{\mathrm{CS}}}=$ $\delta_{j k} \gamma_{k}+\beta / g \Delta_{j k}$, Furthermore, since there is an ambiguity in $\overline{\mathrm{MS}} \rightarrow \overline{\mathrm{CS}}$ rotation matrix, by judicious choice one can "push" mixing under evolution to NNLO. Hence, in $\overline{\mathrm{CS}}$ scheme, we are using, the unknown correction $\Delta_{j k}$ starts at NNLO and it can be additionally suppressed by the choice of an appropriate initial condition. Finally, we express our result in $\overline{\mathrm{CS}}$ scheme as

$$
C_{j}^{\overline{\mathrm{CS}}}=\sum_{k=j}^{\infty} C_{k}\left(\alpha_{s}(\mathcal{Q})\right) \mathcal{P} \exp \left\{\int_{\mathcal{Q}}^{\mu} \frac{d \mu^{\prime}}{\mu^{\prime}}\left[\gamma_{j}\left(\alpha_{s}\left(\mu^{\prime}\right)\right) \delta_{k j}+\frac{\beta}{g} \Delta_{k j}\left(\alpha_{s}\left(\mu^{\prime}\right)\right)\right]\right\},
$$

with $C_{k}\left(\alpha_{s}(\mathcal{Q})\right)$ obtained from the $\eta=\xi$ limit of Eq. (8) and using $c_{j}\left(\alpha_{s}\right)$. As stated above the $\Delta_{k j}$ mixing term appears at NNLO and is neglected. We take $c_{j}$ and $\gamma_{j}$ calculated to NNLO order from Refs. [11], and obtain the DVCS prediction to NNLO in the $\overline{\mathrm{CS}}$ scheme.

\section{Results}

We have used the formalism described in the preceding sections to investigate the size of NNLO corrections to non-singlet [6] and singlet Compton form factors [7], to obtain complete (non- 
diagonal evolution included) $\overline{\mathrm{MS}}$ NLO predictions [5], and to perform fits, in both $\overline{\mathrm{MS}}$ and $\overline{\mathrm{CS}}$ schemes, to DVCS and DIS data and extract information about GPDs [5].

One can use a simple Regge-inspired ansatz for GPDs

$$
\boldsymbol{H}_{j}\left(\eta, \Delta^{2}, \mathcal{Q}_{0}^{2}\right)=\left(\begin{array}{c}
N_{\Sigma}^{\prime} F_{\Sigma}\left(\Delta^{2}\right) B\left(1+j-\alpha_{\Sigma}(0), 8\right) \\
N_{G}^{\prime} F_{G}\left(\Delta^{2}\right) B\left(1+j-\alpha_{G}(0), 6\right)
\end{array}\right)+\mathcal{O}\left(\eta^{2}\right)
$$

with ( $p_{a}$ is a flavour dependent integer)

$$
\alpha_{a}\left(\Delta^{2}\right)=\alpha_{a}(0)+0.15 \Delta^{2}, \quad F_{a}\left(\Delta^{2}\right)=\frac{j+1-\alpha_{a}(0)}{j+1-\alpha_{a}\left(\Delta^{2}\right)}\left(1-\frac{\Delta^{2}}{M_{0}^{a 2}}\right)^{-p_{a}}
$$

In the forward case $(\Delta=0)$ this ansatz is equivalent to the standard building blocks for PDFs: $\Sigma(x)=N_{\Sigma}^{\prime} x^{-\alpha_{\Sigma}(0)}(1-x)^{7}, G(x)=N_{G}^{\prime} x^{-\alpha_{G}(0)}(1-x)^{5}$. We have performed the analysis of radiative corrections with generic parameters and made fits of parameters $N_{\Sigma}, \alpha_{\Sigma}(0), M_{0}^{\Sigma}, N_{G}$, $\alpha_{G}(0), M_{0}^{G}$. The work on a more realistic $\eta$-dependent ansatz is in progress.

We introduce now the quantities that we utilize as a measures of the scheme dependence and, foremostly, as indicators for the convergence of the perturbation series. It is natural to employ for this purpose the ratios of Compton form factors, i.e., the corresponding modulus and phase difference, at order $\mathrm{N}^{P} \mathrm{LO}$ to the one at order $\mathrm{N}^{P-1} \mathrm{LO}$, where $P=\{0,1,2\}$ stands for LO, NLO, and NNLO order, respectively:

$$
\delta^{P} K=\frac{\left|\mathcal{H}^{\mathrm{N}^{P} \mathrm{LO}}\right|}{\left|\mathcal{H}^{\mathrm{N}^{P-1} \mathrm{LO}}\right|}-1, \quad \delta^{P} \varphi=\arg \left(\frac{\mathcal{H}^{\mathrm{N}^{P} \mathrm{LO}}}{\mathcal{H}^{\mathrm{N}^{P-1} \mathrm{LO}}}\right) .
$$

The phase differences are small, and we will not comment on them here further. The NLO corrections to the moduli in $\overline{\mathrm{MS}}$ and $\overline{\mathrm{CS}}$ schemes have a similar $\xi$-shape, where $\overline{\mathrm{MS}}$ corrections are generally larger. The relative NLO and NNLO corrections in $\overline{C S}$ scheme are depicted in Fig. 2. From the left panel, showing corrections at the input scale, we realize that the large negative NLO corrections to the modulus (thick dashed) in the 'hard' gluon scenario are shrunk at NNLO to less than $10 \%$ (thick solid), in particular in the small $\xi$ region. In the 'soft' gluon case the NNLO corrections (thin solid) are $\pm 5 \%$. For $\xi \sim 0.5$, the corrections are reduced only unessentially and are around $5 \%$ and $10 \%$ at both NLO and NNLO level. If evolution is switched on (right panel), our findings drastically change. For $\xi \gtrsim 5 \cdot 10^{-2}$ NNLO corrections are stabilized on the level of about $3 \%$ at $\mathcal{Q}^{2}=100 \mathrm{GeV}^{2}$. But they start to grow with decreasing $\xi$ and reach at $\xi \approx 10^{-5}$ the $20 \%$ level. As in DIS, this breakdown of perturbation theory at small $\xi$ obviously stems from evolution and is thus universal, i.e., process independent. The large change of the scaling prediction within the considered order does not influence the quality of fits, and, in particular, the possibility of relating DVCS and DIS data. Hence, the problem of treatment or resummation of these large corrections is relevant primarily to our partonic interpretation of the nucleon content. As long as we precisely define the treatment of the evolution operator, perturbative QCD can be employed as a tool for analyzing data also in the small $\xi$ region.

The Mellin-Barnes integral approach offers the possibility for a fast and numerically stable analysis. Our numerical routine is designed for the purpose of fitting DVCS (and DIS) observables [12] and testing various GPD ansaetze; an example is presented in Fig. 3. 

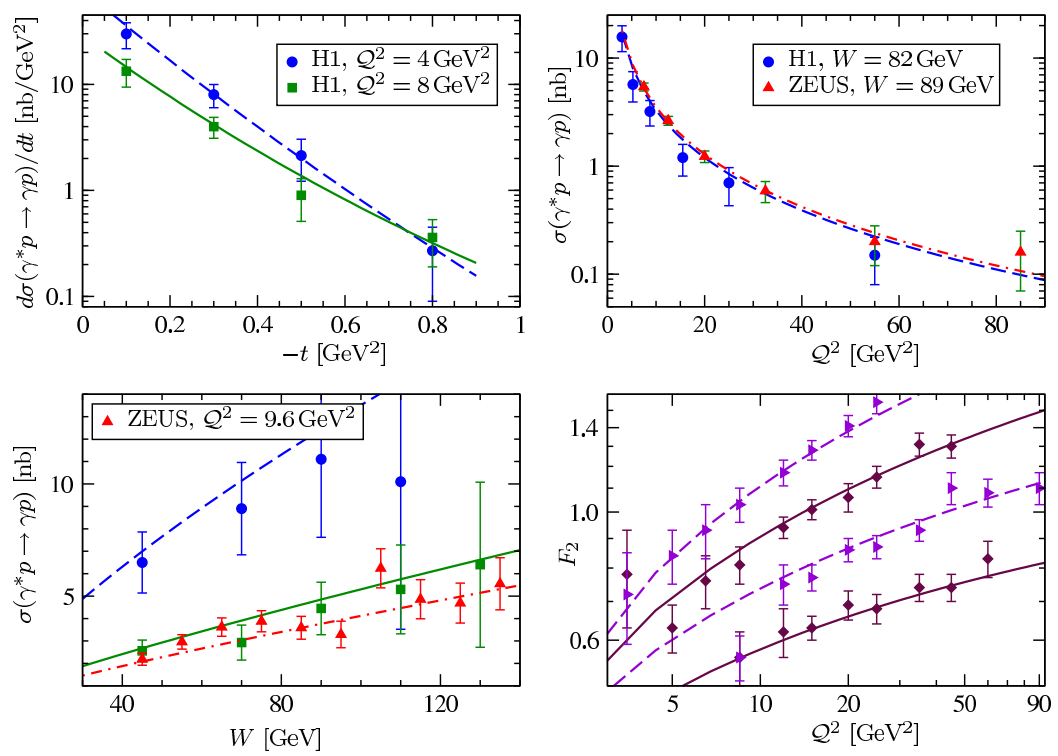

Fig. 3: Fit example: differential DVCS cross section versus $\Delta^{2}$ (up,left), $\Delta^{2}$-integrated cross section versus $\mathcal{Q}^{2}$ (up,right) and $W$ (down,left) as well as the DIS structure function $F_{2}$ at $x_{b}=8 \times 10^{-3}, 3.2 \times 10^{-3}, 1.3 \times 10^{-3}, 4 \times$ $10^{-4}$ (down,right).

Fourier transform of resulting GPDs for $\eta=0$,

$$
H(x, \mathbf{b})=\int \frac{d^{2} \boldsymbol{\Delta}}{(2 \pi)^{2}} e^{-i \mathbf{b} \cdot \boldsymbol{\Delta}} H\left(x, \eta=0, \Delta^{2}=-\boldsymbol{\Delta}^{2}\right),
$$

can be interpreted in the infinite momentum frame as probability density [13], see Fig. 4b. The average transversal parton distance squared $\left\langle\mathbf{b}^{2}\right\rangle$ is given by the GPD slope $B=\left\langle\mathbf{b}^{2}\right\rangle / 4$, shown in Fig. 4a. The results confirm the picture, mentioned in the introduction, about the correlation of transversal and longitudinal degrees of freedom: harder partons are closer to the center.

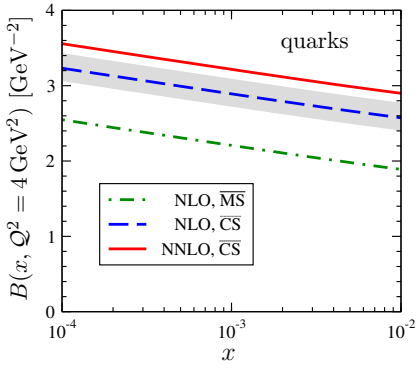

a)

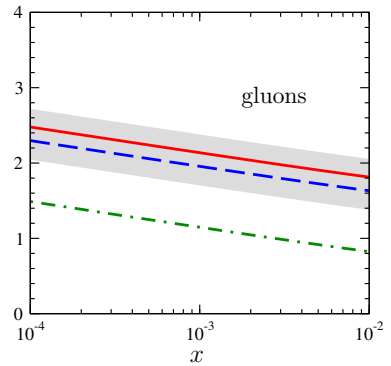

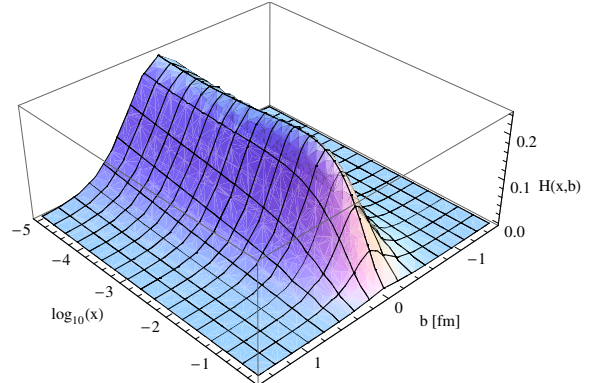

b)

Fig. 4: a) Resulting GPD slope $B=\left\langle\mathbf{b}^{2}\right\rangle / 4$ at the input scale $\mathcal{Q}^{2}=4 \mathrm{GeV}^{2}$ and b) 3D picture of gluon GPD (13). 


\section{Summary}

GPDs encode a unified description of the proton structure and they are experimentally accessible via the hard leptoproduction of photon or mesons. We have shown that the representation of Compton form factors as Mellin-Barnes integrals offers a useful tool in analyzing DVCS: the inclusion of evolution is simple, numerical treatment is stable and fast. Although the motivation for this representation originated from manifest conformal symmetry at LO, we have shown here that this Mellin-Barnes integral representation can be used within the standard $\overline{\mathrm{MS}}$ scheme beyond LO. Such a representation can straightforwardly be obtained from the momentum fraction representation and, therefore, also other GPD related processes, e.g., the hard electroproduction of mesons, can be given in terms of Mellin-Barnes integrals. This opens a new road for the 'global' analysis of experimental data within the perturbative GPD formalism to NLO accuracy. Furthermore, the use of conformal symmetry enables elegant approach to higher-order radiative corrections to the DVCS amplitude. We have shown that although NLO corrections can be sizable, and are strongly dependent on the gluonic input, the NNLO corrections are small to moderate, supporting perturbative framework of DVCS. The observed change in the scale dependence is not so conclusive: similarly as in DIS we encounter large NNLO effects for $\xi<10^{-3}$, which signal a breakdown of naive perturbation series in the evolution operator. Nevertheless, this breakdown is universal and if we precisely define the treatment of this operator, perturbative QCD can be employed as a tool for analyzing data even in the small $\xi$ region. Finally, fits to available DVCS and DIS data work well and give access to transversal distribution of partons.

\section{References}

[1] D. Müller, D. Robaschik, B. Geyer, F.-M. Dittes, and J. Hořejši, Fortschr. Phys. 42, 101 (1994); A. Radyushkin, Phys. Lett. B380, 417 (1996). hep-ph/9604317.

[2] X.-D. Ji, Phys. Rev. Lett. 78, 610 (1997). hep-ph/9603249.

[3] M. Diehl, Phys. Rept. 388, 41 (2003). hep-ph/ 0307382 ; A. V. Belitsky and A. V. Radyushkin, Phys. Rept. 418, 1 (2005). hep-ph / 0504030.

[4] D. Müller and A. Schäfer, Nucl. Phys. B739, 1 (2006). hep-ph/ 0509204.

[5] K. Kumerički, D. Müller, and K. Passek-Kumerički (2007). hep-ph / 0703179.

[6] D. Müller, Phys. Lett. B634, 227 (2006). hep-ph / 0510109.

[7] K. Kumerički, D. Müller, K. Passek-Kumerički, and A. Schäfer, Phys. Lett. B648, 186 (2007). hep-ph/0605237.

[8] X.-D. Ji and J. Osborne, Phys. Rev. D57, 1337 (1998). hep-ph/9707254; A. V. Belitsky and D. Müller, Phys. Lett. B417, 129 (1998). hep-ph/9709379; L. Mankiewicz, G. Piller, E. Stein, M. Vanttinen, and T. Weigl, Phys. Lett. B425, 186 (1998). hep-ph/9712251.

[9] D. Müller, Phys. Rev. D49, 2525 (1994);

A. V. Belitsky and D. Müller, Nucl. Phys. B527, 207 (1998). hep-ph / 9802411 ;

A. V. Belitsky and D. Müller, Nucl. Phys. B537, 397 (1999). hep-ph/ 9804379.

[10] D. Müller, Phys. Rev. D58, 054005 (1998). hep-ph/ 9704406 ;

D. Müller, Phys. Rev. D59, 116003 (1999). hep-ph/9812490;

B. Melić, D. Müller, and K. Passek-Kumerički, Phys. Rev. D68, 014013 (2003). hep-ph/ 0212346.

[11] E. B. Zijlstra and W. L. van Neerven, Nucl. Phys. B383, 525 (1992); A. Vogt, S. Moch, and J. A. M. Vermaseren, Nucl. Phys. B691, 129 (2004). hep-ph / 0404111. 
[12] H1 Collaboration, C. Adloff et al., Phys. Lett. B517, 47 (2001). hep-ex/ 0107005 ; ZEUS Collaboration, S. Chekanov et al., Phys. Lett. B573, 46 (2003). hep-ex/ 0305028 ; H1 Collaboration, A. Aktas et al., Eur. Phys. J. C44, 1 (2005). hep-ex/ 0505061.

[13] M. Burkardt, Phys. Rev. D62, 071503 (2000). hep-ph / 0005108 ; M. Burkardt, Int. J. Mod. Phys. A18, 173 (2003). hep-ph/ 0207047. 